University of Nebraska - Lincoln

DigitalCommons@University of Nebraska - Lincoln

Peter Dowben Publications

Research Papers in Physics and Astronomy

September 1995

\title{
A novel design for a small retractable cylindrical mirror analyzer
}

D.N. Mcllroy

University of Nebraska-Lincoln

Peter A. Dowben

University of Nebraska-Lincoln, pdowben@unl.edu

A. Knop

Institut für Physikalische und Theoretische Chemie, Freie Universitat Berlin

E. Ruhl

Institut für Physikalische und Theoretische Chemie, Freie Universitat Berlin

Follow this and additional works at: https://digitalcommons.unl.edu/physicsdowben

Part of the Physics Commons

Mcllroy, D.N.; Dowben, Peter A.; Knop, A.; and Ruhl, E., "A novel design for a small retractable cylindrical mirror analyzer" (1995). Peter Dowben Publications. 122.

https://digitalcommons.unl.edu/physicsdowben/122

This Article is brought to you for free and open access by the Research Papers in Physics and Astronomy at DigitalCommons@University of Nebraska - Lincoln. It has been accepted for inclusion in Peter Dowben Publications by an authorized administrator of DigitalCommons@University of Nebraska - Lincoln. 


\title{
SHOP NOTES
}

These are "how to do it" papers. They should be written and illustrated so that the reader may easily follow whatever

instruction or advice is being given.

\section{A novel design for a small retractable cylindrical mirror analyzer}

\author{
D. N. Mcllroy and P. A. Dowben \\ Department of Physics and Astronomy, Behlen Laboratory of Physics, University of Nebraska-Lincoln, \\ Lincoln, Nebraska 68588-0111
}

A. Knop and E. Rühl

Institut für Physikalische und Theoretische Chemie, Freie Universität Berlin, Takustr. 3, D-14195 Berlin, Germany

(Received 20 January 1995; accepted 24 June 1995)

\begin{abstract}
In this paper we will review the performance of a "miniature" single pass cylindrical mirror analyzer (CMA) which we have used successfully in a variety of experiments. The underlying premise behind this CMA design was to minimize spatial requirements while maintaining an acceptable level of instrumental resolution. While we are presenting the results of a single pass cylindrical mirror analyzer, improvements on the present design, such as going to a double pass design, will undoubtedly improve the instrumental resolution. () 1995 American Vacuum Society.
\end{abstract}

The electro-optics of the CMA is a modification from a standard design by Lee and Pethica ${ }^{1}$ derived from standard electrostatic relationships, ${ }^{2-5}$ where the only design restriction is that the inner cylinder is held at ground. The CMA is designed around an optimal acceptance angle $\left(\theta_{0}\right)$ of $42^{\circ}$ $18.5^{\prime}, 6$ with the focus approximately $6 \mathrm{~mm}$ in front of the entrance slit of the inner cylinder. The overall dimensions of the electro-optics are approximately $30 \mathrm{~mm}$ in diameter and $45 \mathrm{~mm}$ in length. The outer shell of the CMA is constructed of $\mathrm{Mu}$ metal to minimize resolution broadening due to stray magnetic fields. A simple commercial channeltron is employed for electron detection. In Fig. 1 a basic schematic of the CMA design is shown. The control scheme of the CMA illustrated in Fig. 2 is only one of many ways that the control electronics can be configured.

In Fig. 3 we present the differentiated spectrum of the elastic peak of electrons reflected off of the (100) surface of a flat nickel single crystal. The sample area was approximately $12 \mathrm{~mm}^{2}$ (approximately the size of the electron beam) and the sample current during data acquisition was $10 \mu \mathrm{A}$. From this spectrum we see that the instrumental linewidth of the elastic peak is quite good and that the instrumental energy resolution $\left(R=\Delta E / E_{0}\right)$ of the analyzer is $1.5 \%$. An instrumental asymmetry in the signal is introduced by the analyzer as a consequence of inhomogeneities in the fields near the exit aperture and due to the elimination of conducting mesh over the entrance and exit slits in an effort to increase electron transmission. This asymmetry is most apparent in the derivative of the elastic peak in Fig. 3.

The CMA has been constructed to fit onto a linear motion feedthrough mounted on a 2.75 in. conflat flange. The advantages of this design are simple, but highly attractive. The placement of the CMA on a linear motion feedthrough eliminates restraints on chamber designs that are otherwise imposed by the focusing requirements of conventional fixed
CMA detectors. This has the added advantage that it reduces overcrowding, which often occurs in many experimental systems. By mounting the CMA onto a 2.75 in. flange, the analyzer can easily be moved to different locations within a typical ultrahigh vacuum (UHV) chamber. For Auger electron spectroscopy off of a crystal surface, the analyzer was controlled as shown schematically in Fig. 2.

In addition to successfully acquiring Auger spectra from single crystals, we have also obtained Auger electron spectra of atomic Ar beams in a cross beam experiment. It is from this experiment that the true versatility of the CMA design becomes most evident. The experiment consisted of crossing tunable ionizing light from a synchrotron light source with an Ar beam. The photon energy is scanned across an energy range where Ar Auger electron yields are expected, while the CMA is held at a constant pass energy, which in this case corresponded to the kinetic energy of the Ar LMM Auger process. The specifics of the experiment are discussed in greater detail elsewhere. ${ }^{7}$ We have also used this CMA in constant final state spectroscopy and characteristic energy loss spectroscopy experiments.

In Fig. 4 we present Auger spectra of the atomic Ar LMM Auger process acquired at three different photon energies. The broad feature centered at a kinetic energy of approximately $210 \mathrm{eV}$ in the three spectra corresponds to the $\mathrm{Ar}$ Auger electrons, while the feature at approximately 120 and $150 \mathrm{eV}$ in the spectra acquired at photon energies of 370 and $400 \mathrm{eV}$, respectively, originate from $\operatorname{Ar} 2 p$ core electron excitations. The limitations of the CMA in this acquisition mode are apparent from the poor resolution of the Auger spectra in Fig. 4. In reality, the broad Auger feature is composed of numerous individual Auger processes which are not resolvable in our spectra, but have been resolved by Akela et al. ${ }^{8}$ using a larger diameter CMA.

We have attributed the poor resolution of the spectra in 


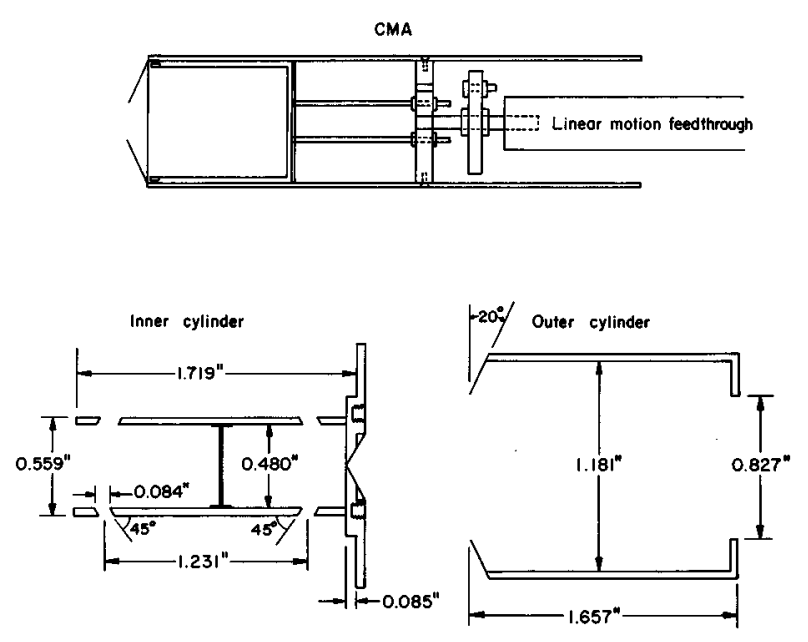

FIG. 1. A schematic drawing of the cylindrical mirror analyzer. The illustration at the top of the figure is an overview of the construction of the analyzer and the two illustrations at the bottom are of the inner and outer cylinders of the analyzer.

Fig. 4 to sample volume effects. In the case where the signal originates from the surface of a solid, the sampling volume is no more than a few angstroms below the surface. Therefore, the focus of the CMA, relative to the sample surface, is well defined. This is not the case for the atomic beam experiments. The sampling volume is the volume of the region where the atomic beam and the light source cross, which in

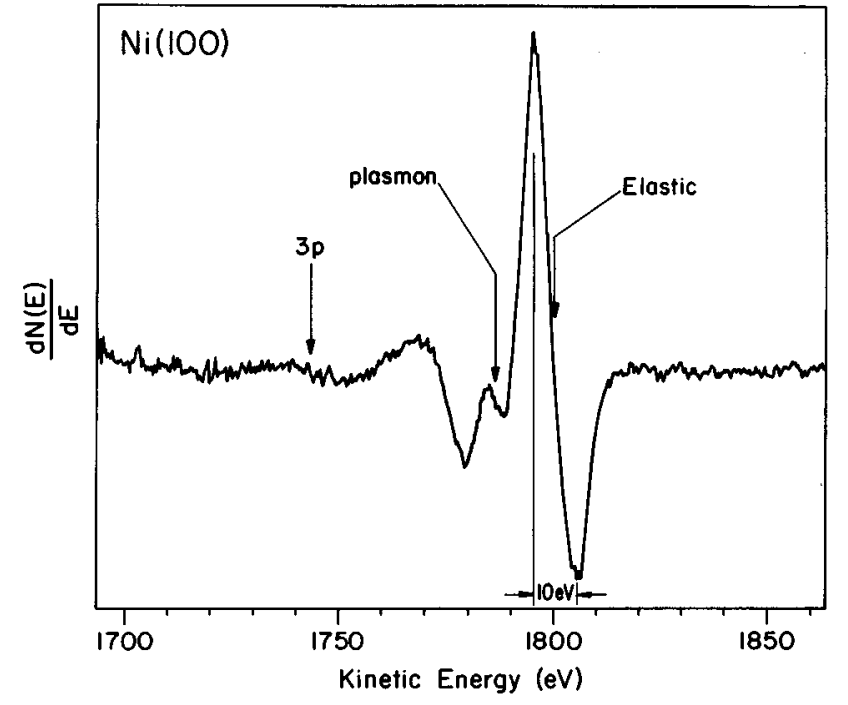

FIG. 3. A spectrum of the elastic peak of electrons reflected off of the (100) surface of a nickel single crystal. An instrumental energy resolution $\left(R=\Delta E / E_{0}\right)$ for the CMA of $1.5 \%$ has been established from the width of the elastic peak. The sample area was approximately $12 \mathrm{~mm}^{2}$ (effectively the size of the electron beam) and the sample current during data acquisition was $10 \mu \mathrm{A}$. The spectrum acquisition time was approximately $2 \mathrm{~min}$.

this experiment was approximately $30 \mathrm{~mm}^{3}$. As a consequence, the focal point of the CMA, relative to the sampling volume, is no longer well defined, i.e., a point source approximation is no longer valid. This has the effect of broad-

CMA CONTROL UNIT

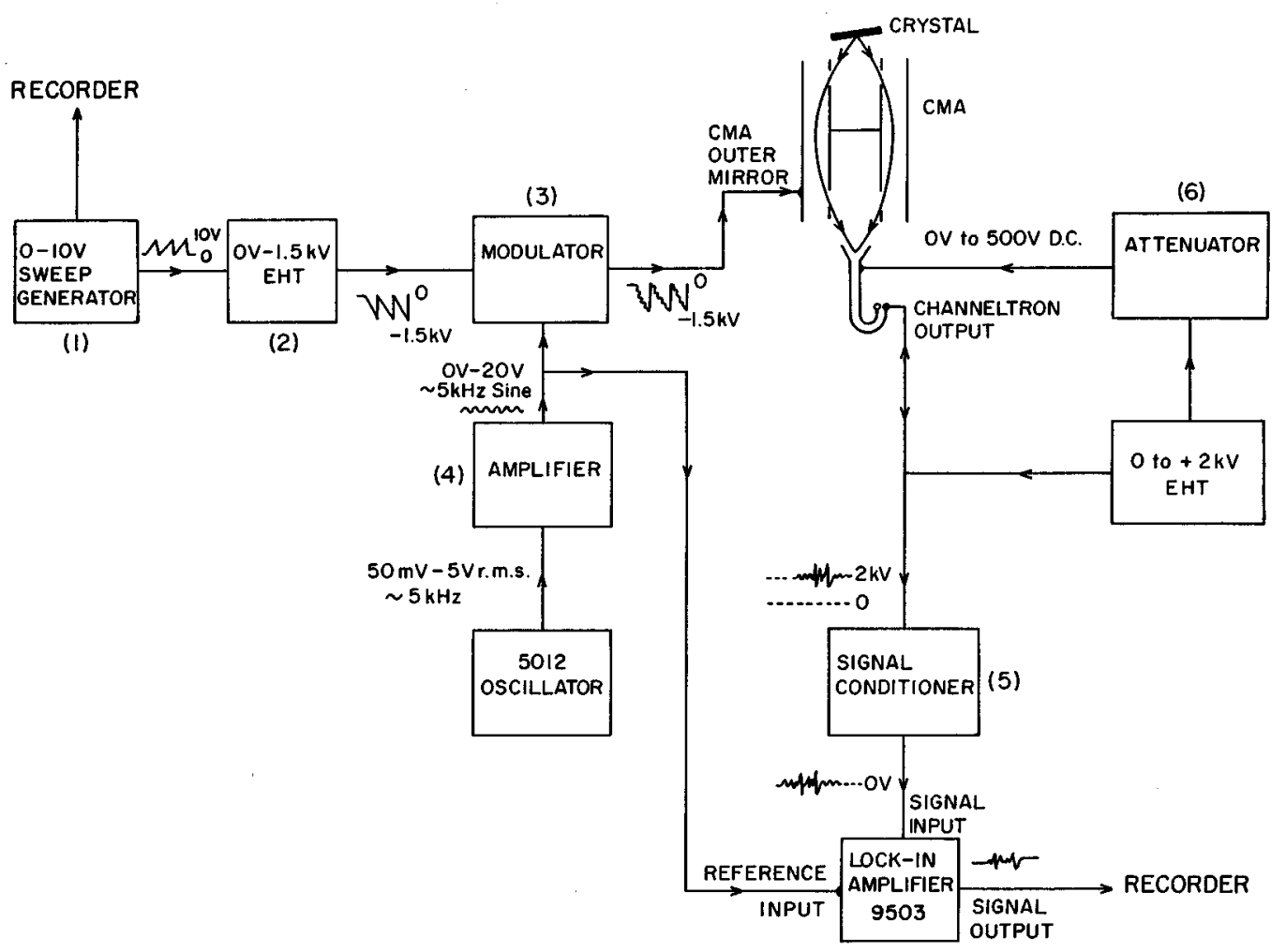

FIG. 2. A flow chart of the control electronics when the analyzer is operated in a lock-in mode, for example, when acquiring Auger spectra. 


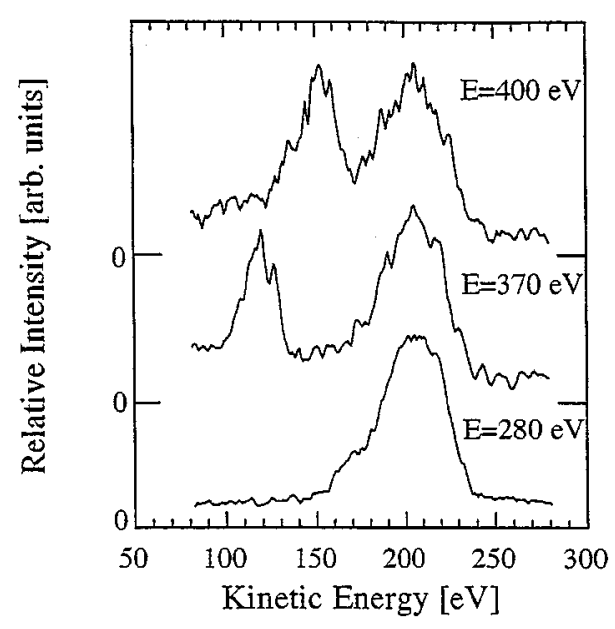

FIG. 4. Auger electron spectra of the atomic Ar $L M M$ process acquired at photon energies of 280, 370, and $400 \mathrm{eV}$. The photon energy independent feature is the $L M M$ Auger process, while the lower kinetic energy feature, which appears in the spectra acquired at photon energies of 370 and $400 \mathrm{eV}$, is the $\operatorname{Ar} 2 p$ excitation. The sampling volume (the volume where the synchrotron light beam and the argon cluster beam crossed) is estimated to be approximately $30 \mathrm{~mm}^{3}$.

ening spectral features which degrades the instrumental resolution of the CMA.

In conclusion, we have designed and built a small cylindrical mirror analyzer which has demonstrated good energy resolution and versatility. By mounting the CMA on a 2.75 in. conflat flange, in conjunction with a linear motion feedthrough, we have been able to utilize it in a variety of configurations and applications. From the elastic peak of an electron beam reflected off of a single crystal of nickel, we have determined the resolution $\left(R=\Delta E / E_{0}\right)$ of the CMA to be $1.5 \%$. From atom cross beam experiments, we have found that the energy resolution is drastically reduced relative to Auger spectra obtained from the surfaces of single crystals. We have attributed this to the larger sampling volume of cross beam experiments, i.e., the focus of the sample is not well defined. In contrast, by running the analyzer in a constant energy mode, very good LMM Auger electron yield measurements of Ar clusters have been achieved. ${ }^{7}$ While we have not explored the full range of applications for this design, these experiments have demonstrated the versatility and usefulness of this CMA design.

Acknowledgments: The authors would like to acknowledge support by the National Science Foundation (INT9300238 and DMR-92-21655) and the Bundesministerium für Technologie (05 5KEFXB5-TP3). The authors would like to acknowledge the assistance of Anthony Lee and J. B. Pethica in developing the CMA.

\footnotetext{
${ }^{1}$ A. Lee and J. B. Pethica (private communication).

${ }^{2}$ M. Karras, M. Pessa, and S. Aksela, Ann. Acad. Sci. Fennicae A VI 289, 1 (1968).

${ }^{3}$ H. Z. Sar-El, Rev. Sci. Instrum. 41, 561 (1970); 38, 1210 (1967).

${ }^{4}$ S. Aksela, Rev. Sci. Instrum. 42, 810 (1971).

${ }^{5}$ B. Wannberg, Nucl. Instrum. Methods 107, 549 (1973).

${ }^{6}$ A. L. Hughes and V. Rojanski, Phys. Rev. 34, 284 (1929).

${ }^{7}$ A. Knop, D. N. McIlroy, P. A. Dowben, and E. Rühl (to be published).

${ }^{8}$ J. Värynen and S. Aksela, J. Electron. Spectrosc. Relat. Phenom. 16, 423 (1979).
} 